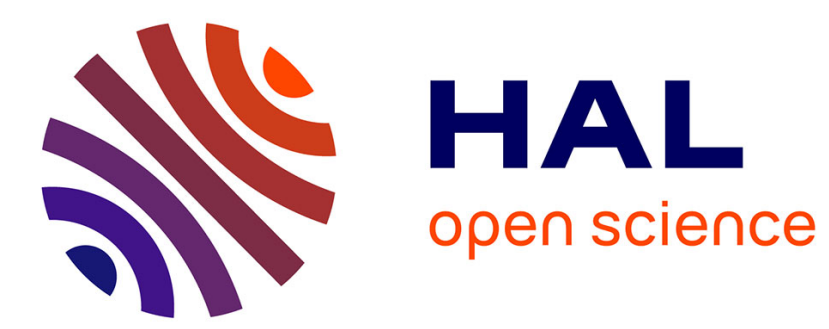

\title{
SIZING, CYCLE TIME AND PLANT CONTROL USING DIOID ALGEBRA
}

Saïd Amari, Isabel Demongodin, Jean Jacques Loiseau

\section{To cite this version:}

Saïd Amari, Isabel Demongodin, Jean Jacques Loiseau. SIZING, CYCLE TIME AND PLANT CONTROL USING DIOID ALGEBRA. A. Dolgui J. Soldek and O. Zaikin (Eds.), Springer. SIZING, CYCLE TIME AND PLANT CONTROL USING DIOID ALGEBRA, Springer, pp.71-85, 2004. hal00362950

\section{HAL Id: hal-00362950 https://hal.science/hal-00362950}

Submitted on 19 Feb 2009

HAL is a multi-disciplinary open access archive for the deposit and dissemination of scientific research documents, whether they are published or not. The documents may come from teaching and research institutions in France or abroad, or from public or private research centers.
L'archive ouverte pluridisciplinaire HAL, est destinée au dépôt et à la diffusion de documents scientifiques de niveau recherche, publiés ou non, émanant des établissements d'enseignement et de recherche français ou étrangers, des laboratoires publics ou privés. 
Chapter 6 in Supply Chain Optimisation, Series Applied Optimization,

A. Dolgui J. Soldek and O. Zaikin (Eds.), Springer. pp. 71-85, 2004

\title{
SIZING, CYCLE TIME AND PLANT CONTROL USING DIOID ALGEBRA
}

\author{
S. Amari, I. Demongodin, J.-J. Loiseau \\ IRCCYN - UMR CNRS n ${ }^{\circ} 6597$ \\ 1, rue de la Nöe - BP 92101 - 44321 Nantes, cedex 03 - France \\ \{amari,demongodin,loiseau\}@irccyn.ec-nantes.fr
}

\begin{abstract}
Using an industrial process from the car sector, we show how dioid algebra may be used for the performance evaluation, sizing, and control of this discrete-event dynamic system. Based on a Petri net model as an event graph, max-plus algebra and min-plus algebra permit to write linear equations of the behavior. From this formalism, the cycle time is determined and an optimal sizing is characterized for a required cyclic behavior. Finally, a strict temporal constraint the system is subject to is reformulated in terms of inequalities that the (min, + ) system should satisfy, and a control law is designed so that the controlled system satisfies the constraint.
\end{abstract}

Key words: Dioid algebra, Petri net, sizing, cycle time, control.

\section{INTRODUCTION}

For efficient design and operation techniques of manufacturing systems, it is well known that methods and tools are needed to model complex material and control flows, to analyze behavior and interactions of manufacturing resources and to predict the performance measures such as productivity, cycle times and work-in-process. Thanks to significant advances over the last decade, discrete-event system techniques have gained maturity and provide a wide spectrum of tools for solving problems encountered in design, supervisory control and performance evaluation of manufacturing systems. Petri nets, queuing networks, dioid algebra, perturbation analysis, formal language theory, state-charts have, among many others, proven techniques for modeling, specification and analysis of complex discrete-event dynamic systems (DEDS). 
The problem tackled here is the performance evaluation, the optimal sizing and the control of a manufacturing plant from the sector of the car industry. The studied process can be embedded in the discrete-event dynamic system class. Among various techniques of analysis and control [2], a theory of linear algebra for DEDS also known as dioid theory has been recently developed [1]. More precisely, as long as the DEDS has no conflict, it can be modeled by an event graph or marked graph, i.e. by a Petri net in which each place has exactly one input and one output transitions. Such a graph is called a Timed Event Graph (TEG) when a sojourn time is associated to each place, or by duality, when a delay is associated to each transition. Hence, the timed behavior of such a TEG is represented by the dates at which the transitions of a TEG are fired for the $k^{\text {th }}$ time or equivalently, by the number of firings of transitions at time $t$. The former description, as daters in the events domain, leads to linear equations in the so-called max-plus algebra, while in the latter, as counters in the time domain, equations are linear in the min-plus algebra. In both cases, there exists a spectral theory that leads to one of the main results in that frameworks concerning performance evaluation of DEDS. The cycle time, or the throughput, is the solution of an eigenvalue problem in the considered algebra. Beside these performance evaluation interests, control problems have been addressed in the context of DEDS using this algebra. In this paper, the first step concerns the cycle time of the industrial plant using the maxplus algebra. Next, for a required cycle time, the sizing and the control of the plant are determined using the min-plus algebra.

The article is organized as follows. Backgrounds concerning the dioid algebra are recalled in Section 2. A brief description of the industrial plant is given in Section 3. A Timed Event Graph model of the plant is provided in Section 4, and linear equations associated to the system in the max-plus dioid algebra are derived in Section 5. On the basis of that model, the cycle time is determined in the next section for a given configuration, that is a given number of available pallets. The sizing problem is tackled in Section 7 , where the number of free pallets is optimized for a required cycle time. Finally a control problem is addressed and solved in Section 8.

\section{DIOID ALGEBRA}

Definition 1 (Dioid algebra).

(i) An abelian monoid is a set $D$ endowed with an internal law $\oplus$ that is associative, commutative, and admits a neutral element denoted $\varepsilon$.

(ii) An abelian semiring is a monoid endowed with a second operation $\otimes$, also associative, distributive with respect to the first law $\oplus$, admitting a 
neutral element $e$, and so that the neutral element of the first law is absorbing for the second law $\otimes$ (i.e. $\varepsilon \otimes a=a \otimes \varepsilon=\varepsilon, \forall a \in D$ ).

(iii) A dioid is a semiring with an idempotent first law (i.e. $a \oplus a=a, \forall a \in D)$.

One says that the dioid is commutative provided that the law $\otimes$ is commutative.

Example 1 (Max-plus algebra). $\bar{\Re}_{\max }=\{\mathfrak{R} \cup\{-\infty\} \cup\{+\infty\}, \max ,+\}$ is a commutative dioid, with zero element $\varepsilon$ equal to $-\infty$, and the unit element $e$ equal to 0 . We adopt the usual notation, so that the symbol $\oplus$ stands for the max operation, and $\otimes$ stands for the addition. Notice that $\varepsilon \otimes+\infty=(-\infty)+(+\infty)=\varepsilon=-\infty$ in $\bar{\Re}_{\max }$.

Example 2 (Min-plus algebra). $\bar{R}_{\min }=\{\mathfrak{R} \cup\{-\infty\} \cup\{+\infty\}, \min ,+\}$ is also a commutative dioid, for which $\varepsilon$ equals to $+\infty$ and $e$ equals to 0 . We shall denote $\oplus$ ' the min operation in the sequel, and the symbol $\otimes$ ' will stand for the addition. Notice that $\varepsilon \otimes-\infty=(+\infty)+(-\infty)=\varepsilon=+\infty$ in $\bar{R}_{\text {min }}$.

Example 3 (Matrix dioid). Let $(D, \oplus, \otimes)$ be a given dioid, and denote $D^{n \times n}$ the set of square $n \times n$ matrices with entries over $D$. The sum and the product over $D$ extend as usually over $D^{n \times n}$ as follows:

$$
(A \oplus B)_{i j}=A_{i j} \oplus B_{i j} \text { and }(A \otimes B)_{i j}=\bigoplus_{k=1}^{n} A_{i k} \oplus B_{k j} .
$$

One can see that $\left(D^{n \times n}, \oplus, \otimes\right)$ is a dioid. The neutral elements for the law $\oplus$ is the matrix the entries of which equal $\varepsilon$, the neutral element for the law $\otimes$ is the matrix the entries of which equal $e$ on the diagonal and $\varepsilon$ outside. Notice that the products of matrices in $\overline{\mathfrak{R}}_{\max }$ and in $\overline{\mathfrak{R}}_{\text {min }}$ are not equal, (and do not equal the usual sum of matrices.)

Definition 2 (Trace). The trace of a square matrix $A \in D^{n \times n}$ is the sum of its diagonal entries, denoted:

$$
\operatorname{tr}(A)=\bigoplus_{i=1}^{n} A_{i i} .
$$

In the max-plus algebra, we shall also use the notation: 


$$
\left(\operatorname{tr}\left(A^{k}\right)\right)^{\frac{1}{k}}=\frac{1}{k}\left(\bigoplus_{i=1}^{n}\left(A^{k}\right)_{i i}\right), \text { where } A^{k}=\underbrace{A \otimes \cdots \otimes A}_{k \text { times }} .
$$

These concepts are useful to model Timed Event Graphs with sojourn delays associated to places. A TEG is an ordinary Petri net where each place has a single input transition and a single output transition. We denote $p_{i j}$ the place from transition $t_{j}$ to transition $t_{i}$. We assume that $n$ is the number of transitions which are upstream and downstream transitions of places. The number of sink transitions, i.e. without output places, is equal to $s$. The number of transitions called source, having no downstream place, is $m$.

Delays or holding times are associated to places of the TEG. Thus the graph is timed on places. The max-plus approach [1] allows us to model with a system of linear inequations the dynamic behavior of such a TEG.

$$
\left\{\begin{array}{l}
x(k+1) \geq A \otimes x(k) \oplus B \otimes u(k+1) \\
y(k) \geq C \otimes x(k)
\end{array}\right.
$$

where the components of the vector $x(k)$ are the firing times of the $n$ transitions $t_{i}$ for the $k^{\text {th }}$ occurrence, the components of $u(k)$ and of $y(k)$ are respectively the firing dates of the source and of the sink transitions. $A, B$ and $C$ are respectively matrices of size $n \times n, n \times m$ and $s \times n$.

\section{PLANT DESCRIPTION}

The process we study here (see reference [6] for more details) is composed of three conveyors belt connected by loops (Figure 1). The parts are made on an extruding machine on loop 3. Loops 1 and 2 are both similar one to each other; they are dedicated to a thermal processing of the parts. Loop 3 processes parts that are conveyed on pallets to one of the other loops. On loop 1, respectively on loop 2, pallets are devoted for a certain type of parts coming from loop 3. Hence, synchronization is needed between these three transfer elements. The machine on loop 3 is very flexible and therefore can provide any type of part needed on loop 1 or loop 2 . The main problem is to achieve the thermal treatment on loop 1 or loop 2 without major failures. 


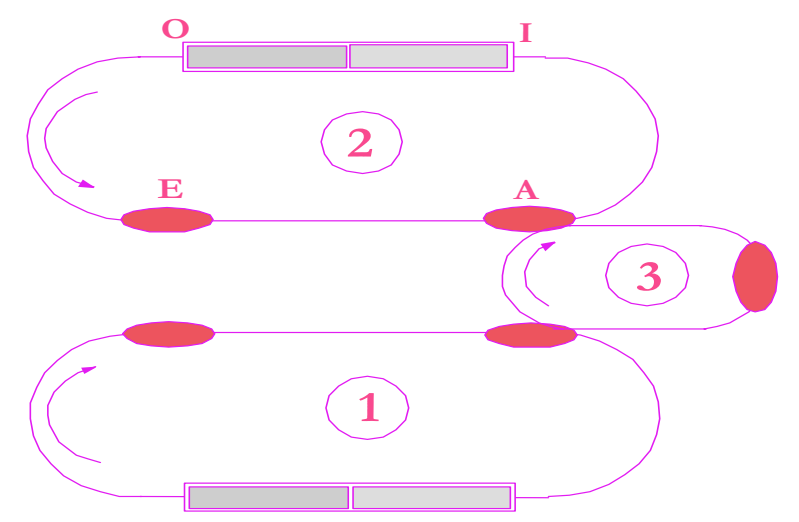

Figure 1: The plant.

Assume loop 2 is under study (identical process for loop 1) (see Figure 2). Parts arrive (from loop 3 ) at point $A$ and an operator fixes them to a pallet. The pallets are then released on a conveyor belt that leads them to point I. Here they enter inside the furnace. This element is a channel divided into two sections. Inside the former section parts are heated and they are next cooled down inside the latter. We can remark that there is no buffer between these two sections. Once pallets come outside the furnace (point $\mathrm{O}$ ), they are transferred to a second operator who removes parts from the pallets. Thus, parts are taken away at point E according to the external resources. Finally, the free pallets are released and transfer to point A.

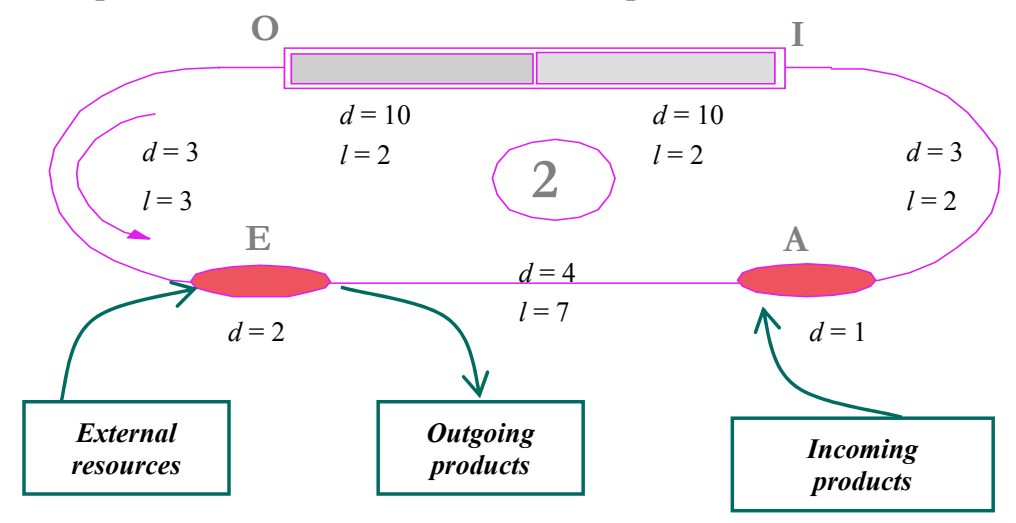

Figure 2: Loop 2.

The constraint for the thermal processing is that no overrun on the heating time is permitted. If so, all parts are rejected and the whole process has to be restarted. The problem is that from time to time, parts are not taken away immediately and those perturbations can affect the production achievement if the sizing, in number of pallets involved, is not correctly designed. 


\section{TIMED EVENT GRAPH OF THE LOOP}

Let us consider loop 2. The capacity of conveyor E-A is equal to 7 pallets, while 5 pallets are free on this conveyor. This physical process is modeled thanks to a TEG with sojourn delays associated to places. The durations of operations (noted $d$ in Figure 2) are assumed to be identical for all the parts and are reported beside places on the Petri net model (see Figure 3 ). For instance, the transfer time from point $\mathrm{E}$ to point $\mathrm{A}$ is considered to be equal to four time units and is represented by place $p_{17}$ (i.e. place from transition $t_{7}$ to transition $t_{1}$ ). Tokens represent the resources of the plant: the pallets, the operators or the conveyor capacities (noted $l$ in Figure 2). Transition $t_{1}$ models the beginning of the fixing operation and transition $t_{2}$ the end of that operation. Only one token is available for this firing transition, meaning that the operator can treat one part at a time. The delay spent for this task is equal to one time unit. The beginning of the thermal process, point $\mathrm{I}$, is modeled by the firing of transition $t_{3}$.

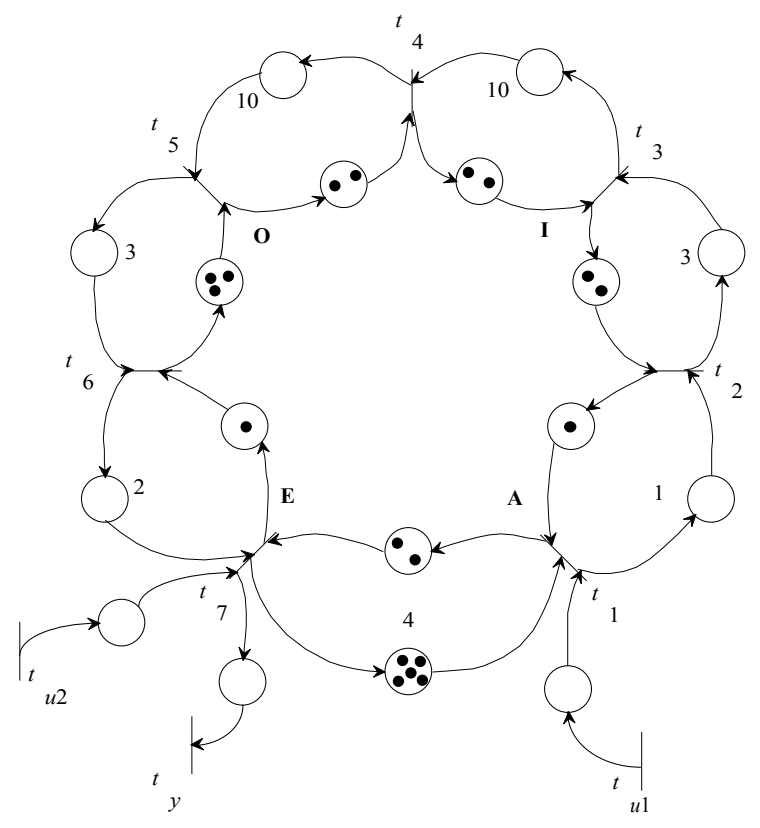

Figure 3: Model of the plant - loop 2.

Transition $t_{y}$ represents the departure of an achieved part, $t_{u 2}$ models the necessity of a resource to carry the terminated part and $t_{u 1}$ models parts arrivals from loop 3. Figure 3 shows the process in an initial state where the five pallets involving through the system are available. The maximal capacity of the conveyor from point $\mathrm{E}$ to point $\mathrm{A}$ is supposed to be equal to seven pallets. 


\section{DIOID MODEL OF THE LOOP}

The construction of matrix $A$, see (1), can be done in several different ways. Olsder et al. have proposed in [7] a method to built max-plus models for large scale systems. For relatively small scale systems, like in the case of this study, one can rather apply the method proposed by Mairesse [5]. That method consists in an addition of some places and transitions in order to obtain a TEG with places containing either zero or one token. A null delay is associated to those additional places. One can see in Figure 4 the resulting TEG, applying that method to the model of Figure 3.

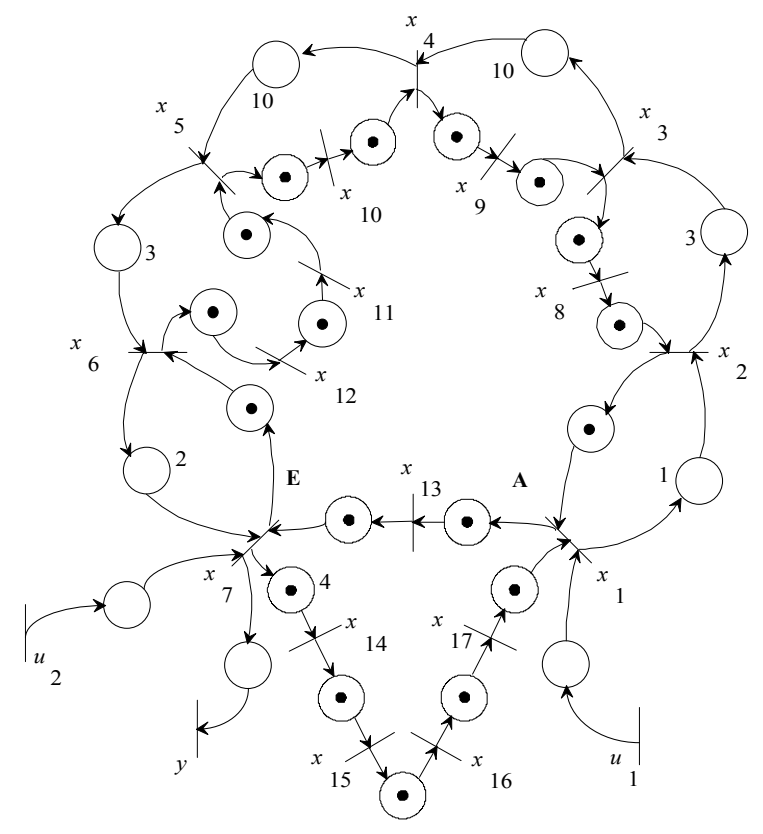

Figure 4: Resulting TEG for loop 2.

The TEG dynamic behavior can therefore be modeled by the following inequations (symbol $\otimes$ is omitted for the seek of readability):

$$
\left\{\begin{array}{l}
x(k+1) \geq A_{0} x(k+1) \oplus A_{1} x(k) \oplus B_{0} u(k+1) \\
y(k) \geq C x(k)
\end{array}\right.
$$

Then, matrices $A_{0}, A_{1}$ and $B_{0}$ are built. $A_{0 i j}$ is the delay associated to the empty place that links transition $t_{j}$ to transition $t_{i}$. $A_{1 i j}$ is the delay associated to the place containing one token that links transition $t_{j}$ to transition $t_{i}$. $B_{0 i j}$ is the delay associated to the empty place that links transition $t_{u j}$ to transition $t_{i}$. If there is no place linking transition $t_{j}$ to transition $t_{i}$ (resp. transition $t_{u j}$ to 
transition $t_{i}$ ) the entry is $\varepsilon$. If the delay is zero, it is noted $e$. Matrices $A_{0}, A_{1}$, $B_{0}$ and $C$ associated to the TEG of Figure 3 are given bellow, where $\varepsilon$ is replaced by a dot for the seek of readability.
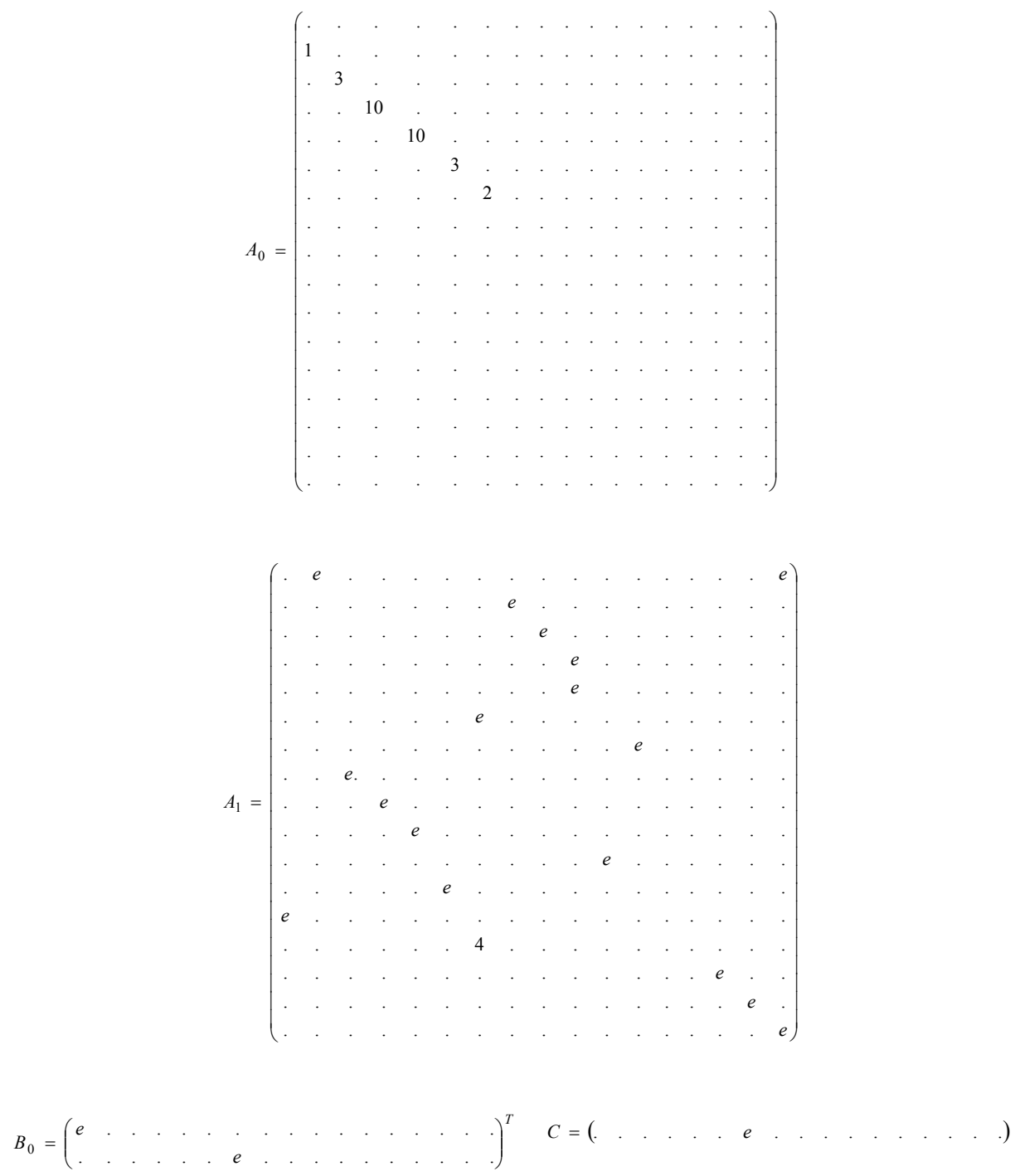

For TEG under maximal speed assumption, system of inequations (2) turns into a system of equations. Furthermore one can derive the system of explicit equations (3):

$$
\left\{\begin{array}{l}
x(k+1)=A x(k) \oplus B u(k+1) \\
y(k)=C x(k)
\end{array}\right.
$$


with matrices $A=A_{0}^{*} A_{1}$ and $B=A_{0}^{*} B_{0}$, where operator * stands for the Kleene star operator: $a^{*}=e \oplus a \oplus a^{2} \oplus \ldots$.
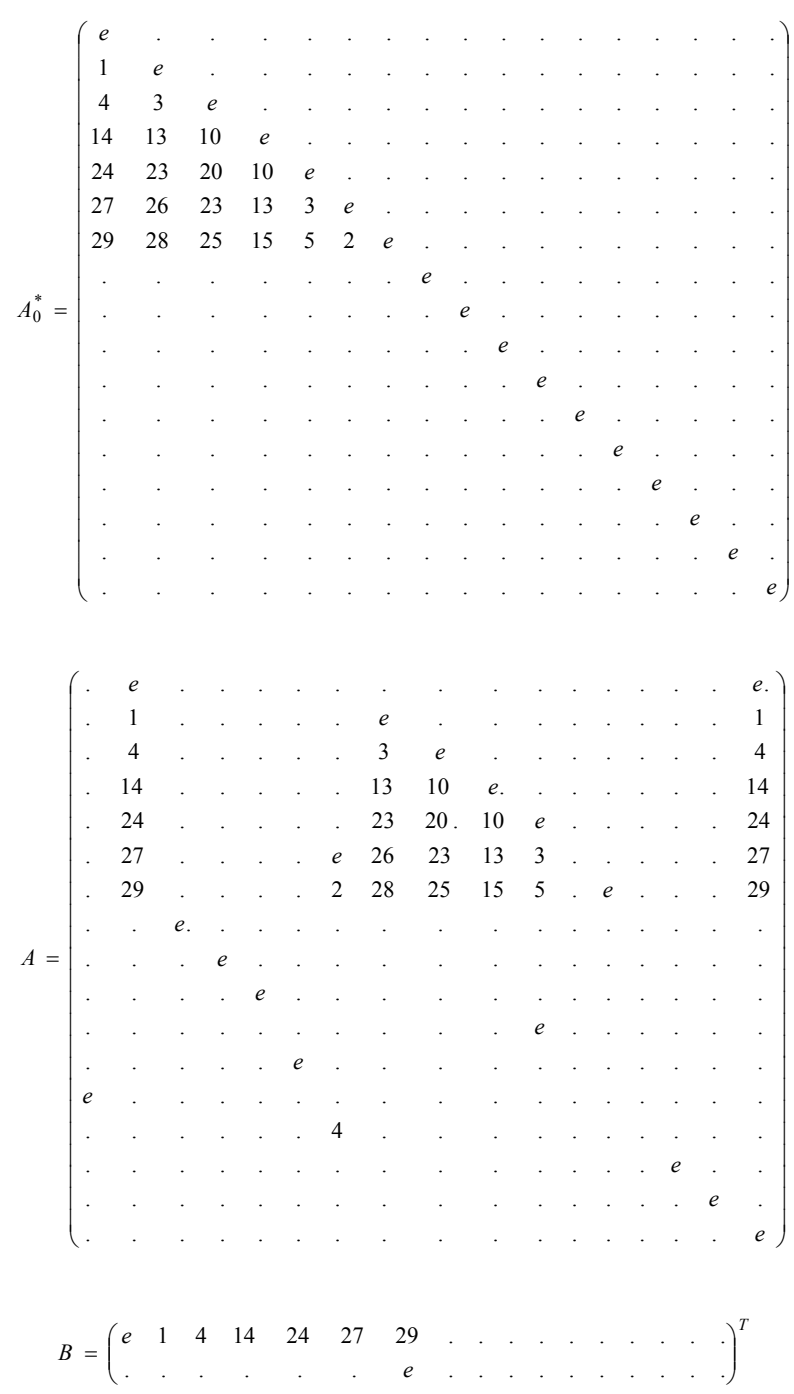

\section{CYCLE TIME OF THE AUTONOMOUS LOOP}

The throughput is maximal if inputs do not constrain the evolution, i.e. if parts are always available at point $\mathrm{A}$ and transportation utilities always present at point E. This is modeled by $u_{i}(k)=\varepsilon$. Then, the system is said to 
be autonomous, without source transitions, and can be modeled by equation (4).

$$
x(k+1)=A x(k)
$$

As long as its graph is strongly connected, or equivalently, matrix $A$ is irreducible, after a finite transient behavior, the system reaches a periodic regime. Therefore, one can write:

$$
\exists K, \forall k \geq K, x(k+c)=\lambda^{c} x(k)
$$

where $\lambda$ refers to equation (6):

$$
A x=\lambda x
$$

and $c$ is the cyclicity of matrix $A$. In other words, $c$ parts are achieved every $c \times \lambda$ time units. The eigenvalue $\lambda$ is unique for a strongly connected event graph and the throughput is given directly as $1 / \lambda$. This eigenvalue is computed according to the algorithm of Cochet-Terrasson et al. [3].

$$
\lambda=\bigoplus_{k=1}^{n}\left(\operatorname{tr} A^{k}\right)^{\frac{1}{k}}
$$

Applying this algorithm to the model of the plant, $\lambda=33 / 5=6.6$ time units.

\section{SIZING FOR A REQUIRED CYCLE TIME}

Now, the capacity of the conveyor from point $\mathrm{E}$ to point $\mathrm{A}$ is not fixed (i.e. place $p_{71}$ is empty in Figure 3). The sizing problem is to determine the minimal number of pallets needed to reach the cycle time of 5 units. This required cycle time corresponds to the bottleneck element, i.e. the furnace, which has a production cycle at 5 time units. In other terms, the minimal initial marking of place $p_{17}$ has to be determined. A method to solve this problem is presented by Gaubert in [4]. After having chosen a desired periodic throughput $\bar{\varphi}$, one can calculate in the min-plus algebra, the initial minimal marking $q$. It is shown that the throughput constraint $\varphi(q) \geq \bar{\varphi}$ is equivalent to the existence of a finite subeigenvector of $\bar{\varphi}$. We denote by $T_{i j}$ the holding time of place $p_{i j}$ and by $N_{i j}$ its initial marking. If place $p_{i j}$ does 
not exist, by convention, $T_{i j}=-\infty$ and $N_{i j}=+\infty$. For the TEG of Figure 3, with an initial marking $q_{71}=0$ and $q_{17}$ unknown, these matrix are defined as follow, where the dot replaces the infinite values:

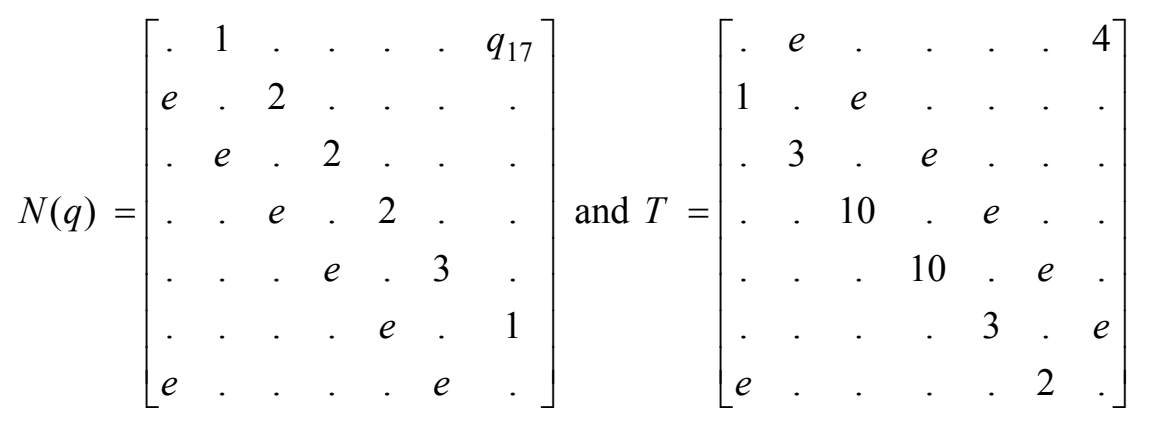

From the method of Gaubert [4], the following assertions are equivalent:

i) $\varphi(q) \geq \bar{\varphi}$

ii) $\rho(C(q)) \geq 0$, with $\left.\rho(C(q))=\bigoplus_{k=1}^{n}\left(\operatorname{tr} C(q)^{k}\right)^{\frac{1}{k}}\right)$

where matrix $C(q)$ is defined by: $C_{i j}(q)=N_{i j}(q)-\bar{\varphi} T_{i j}$.

iii) There exists a vector $\alpha \in \mathfrak{R}^{n}$ such that $\forall i, \min _{j}\left(C_{i j}(q)+\alpha_{j}\right) \geq \alpha_{i}$.

We can notice that $C_{i j}(q)=+\infty$ if either $N_{i j}(q)=+\infty$ or $T_{i j}(q)=-\infty$. This ensures the coherence of the notation 'dot'.

For $\bar{\varphi}=1 / 5, C(q)=N(q)-\frac{1}{5} T$, and thus:

$$
C(q)=\left[\begin{array}{ccccccc}
\cdot & 1 & \cdot & \cdot & \cdot & \cdot & \left(q_{17}-\frac{4}{5}\right) \\
-\frac{1}{5} & \cdot & 2 & \cdot & \cdot & \cdot & \cdot \\
\cdot & -\frac{3}{5} & \cdot & 2 & \cdot & \cdot & \cdot \\
\cdot & \cdot & -\frac{10}{5} & \cdot & 2 & \cdot & \cdot \\
\cdot & \cdot & \cdot & -\frac{10}{5} & \cdot & 3 & \cdot \\
\cdot & \cdot & \cdot & \cdot & -\frac{3}{5} & \cdot & 1 \\
e & \cdot & \cdot & \cdot & \cdot & -\frac{2}{5} & \cdot
\end{array}\right]
$$


Applying assertion ii) with $\bar{\varphi}=1 / 5$, a minimal initial marking of place $p_{17}$ is equal to 7 , as $q_{17} \geq 33 / 5$. Finally, a minimum of 7 free pallets guarantees to the process a cycle time of 5 units.

\section{CONTROL UNDER STRICT TIME CONSTRAINT}

The production unit includes a critical section, which is the warm part of the furnace. The parts should not stay more than 10 time units in this section. Hence the production system is submitted to a strict timed constraint, which is the required sojourn time in the oven. The non respect of the constraint can occur, for instance, because of the lack of transportation resource (point E of Figure 2) during a certain period of time which leads to a blocking of pieces in the furnace. For the normal behavior of the process, the finite product is taken away and the pallets are recycled at the level of point E. In case of a problem at this point, the parts pile up downstream the working station, which may lead to the blocking of parts in the furnace and the cessation of the production.

\subsection{Min-plus model: explicit equation}

To each transition $t_{i}$ we associate the time function $\theta_{i}(t)$, which is the number of firings of transition $t_{i}$ at time $t$. For the TEG of Figure $3, U_{l}(t)$, which is the number of firings of transition $t_{u 1}$ at time $t$, can be seen as a control, which permits to retain the parts arriving from the central loop 3, and postpone their arrival in the production process, if necessary. Similarly, $U_{2}(t)$ can be seen as a disturbance, i.e. the number of firings of transition $t_{u 2}$ at time $t$. In the sequel, $U(t)$ denotes the vector with components $U_{I}(t)$ and $U_{2}(t)$. The min-plus equations of the production unit are deduced from the event graph of Figure 3. They read like follows:

$$
\begin{aligned}
\theta(t)= & A_{0}^{\prime *} A_{1}^{\prime} \theta(t-1) \oplus^{\prime} A_{0}^{\prime *} A_{2}^{\prime} \theta(t-2) \oplus^{\prime} A_{0}^{\prime *} A_{3}^{\prime} \theta(t-3) \oplus^{\prime} A_{0}^{\prime *} A_{4}^{\prime} \theta(t-4) \\
& \oplus^{\prime} A_{0}^{\prime *} A_{10}^{\prime} \theta(t-10) \oplus^{\prime} A_{0}^{\prime *} B^{\prime} U(t)
\end{aligned}
$$

where: 


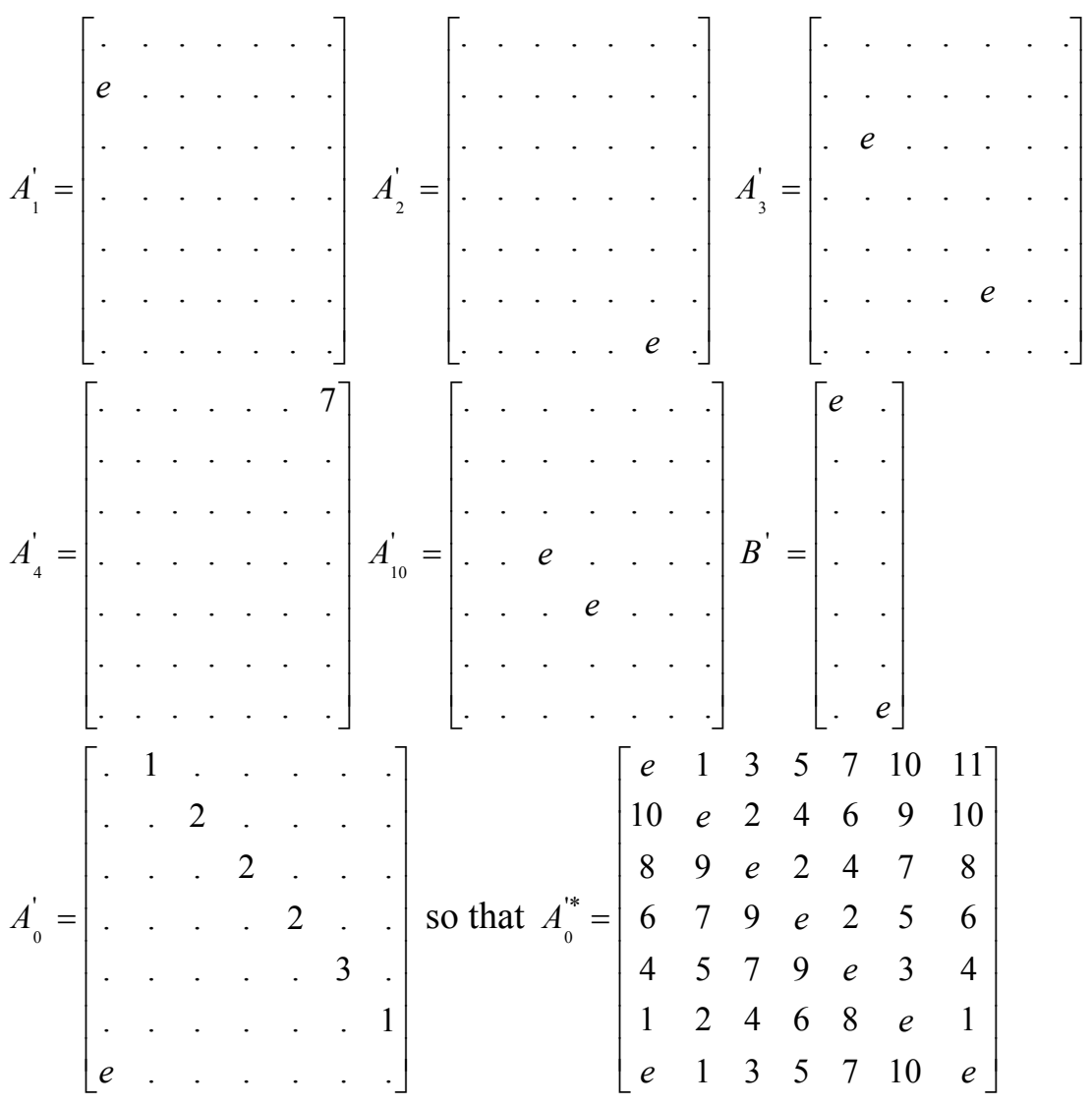

\subsection{Time constraint}

The strict timed constraint holds at the level of the place $p_{43}$ (linking transition $t_{3}$ to transition $t_{4}$ ) which represents the warm part of the furnace. The duration of the thermal treatment is fixed and equals to 10 time units. This constraint can be expressed in terms of the variables $\theta_{i}(t)$, as follows:

$$
\theta_{3}(t)-\theta_{4}(t+10)=0, \forall t \geq 10
$$

The control problem we face consists in synthesizing a control $U_{I}(t)$, knowing $\theta_{i}(t)$ and $U_{2}(t)$, which guarantees the respect of the constraint (9).

Replacing the matrices in the explicit equation (8), one obtains:

$$
\begin{aligned}
& \theta_{4}(t)=7 \theta_{1}(t-1) \oplus^{\prime} 6 \theta_{6}(t-2) \oplus^{\prime} 9 \theta_{2}(t-3) \oplus^{\prime} 5 \theta_{5}(t-3) \\
& \oplus^{\prime} 13 \theta_{7}(t-4) \oplus^{\prime} \theta_{3}(t-10) \oplus^{\prime} 2 \theta_{4}(t-10) \oplus^{\prime} 6 U_{1}(t) \oplus^{\prime} 6 U_{2}(t)
\end{aligned}
$$


from which the following equivalence is deduced:

$$
\theta_{4}(t+10)=\theta_{3}(t) \Leftrightarrow \begin{cases}2 \theta_{4}(t) \geq \theta_{3}(t) & (a) \\ 5 \theta_{5}(t+7) \geq \theta_{3}(t) & (b) \\ 6 \theta_{6}(t+8) \geq \theta_{3}(t) & (c) \\ 13 \theta_{7}(t+6) \geq \theta_{3}(t) & (e) \\ 9 \theta_{2}(t+7) \geq \theta_{3}(t) & (f) \\ 7 \theta_{1}(t+9) \geq \theta_{3}(t) & (g) \\ 6 U_{1}(t+10) \geq \theta_{3}(t) & (h) \\ 6 U_{2}(t+10) \geq \theta_{3}(t) & (i)\end{cases}
$$

One can observe that all these inequalities, except (11-i), readily follow from system (8) of explicit equations. The conclusion is that, provided that (8) holds, the time constraint (9) is satisfied if and only if (11-i) is satisfied.

\subsection{Control synthesis}

Remark that, on the one hand, $U_{2}(t+10) \geq \theta_{7}(t+10)$, which leads to $U_{2}(t+10) \geq \theta_{7}(t)$, since the function $\theta_{7}(t)$ is nondecreasing, and that, on the other hand, $U_{1}(t) \geq \theta_{1}(t) \geq \theta_{2}(t) \geq \theta_{3}(t)$ as system (8) is satisfied. It readily follows that the control law

$$
U_{1}(t)=6 \theta_{7}(t)
$$

implies that the condition (11-i) is satisfied, and consequently that the strict timed constraint is fulfilled.

The control law (12) can be interpreted as a feedback, determining at each instant $t$ the control $U_{l}(t)$ in terms of the state $\theta_{7}(t)$ of the system. This control law is actually a Kanban, which lets a maximum of 6 pallets enter the production loop between point $\mathrm{A}$ and point $\mathrm{E}$ at a time, to avoid any congestion that may be caused by a failure in the working place E. Repeating for the new configuration the computation of the time cycle as in Section 6, one finds that the cycle time value is unchanged and equal to 5 time units. 


\section{CONCLUSION}

We have applied to an industrial process in the car industry, a method for dealing with performance evaluation and sizing based on dioid algebra. First, the system has been specified and modeled as a timed discrete event graph, i.e. a Petri net structure without conflict situation. Next, such model has been dealt with max-plus algebra that provides, through semilinear analysis type methods, an easy way to evaluate the cycle time. Using min-plus algebra, the minimal number of resources required have been determined for an imposed cycle time, and finally a control law has been designed for ensuring the respect of a strict time constraint which exists at the level of a furnace. The control law is optimal since it does not change the requiered cycle time and ensures the robustness of the production.

\section{REFERENCES}

[1] Baccelli F., G. Cohen, G. Olsder, et J. Quadrat. 1992. Synchronization and Linearity: An algebra for Discrete Event Systems. Willey.

[2] Cassandras C.G. and S. Lafortune. 1999. Introduction to discrete event systems. Kluwer Academic.

[3] Cochet-Terrasson J., G.Cohen, S.Gaubert, M.Mc Gettrick, J.P.Quadrat. 1998. 'Numerical computation of spectral elements in max-plus algebra'. IFAC conference on system structure and control, France, pp. 699-706.

[4] Gaubert S. 1995. 'Resource optimization and (min,+) spectral theory'. IEEE trans. on automatic control, 40(11), pp. 1931-1934.

[5] Mairesse J. 1998. 'Petri nets, (max,+) algebra and scheduling'. Actes de

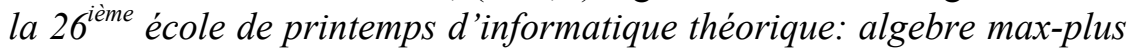
et applications en informatique et automatique, INRIA, LIAFA, IRCyN, France, pp. 329-357.

[6] Martinez C. and P. Castagna. 2003. 'Sizing of an industrial plant using tight time constraints using complementary approaches: $(\max ,+)$ algebra and computer simulation'. Simulation Modelling Practice and Theory, Elsevier, vol. 11, pp. 75-88.

[7] Olsder G.J., Subiono, M.Mc Guettrick. 1998. 'On large scale max-plus algebra model in railway systems'. Actes de la $26^{\text {ième }}$ école de printemps d'informatique théorique: algebre max-plus et applications en informatique et automatique, INRIA, LIAFA, IRCyN, France, pp. 177192. 